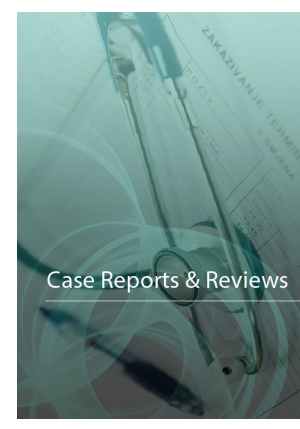

Correspondence

Nathaniel Parker

Kansas University School of Medicine, 1010 N Kansas St, Wichita, KS 67214, USA

E-mail: nparker6@kumc.edu

- Received Date: 12 Oct 2019;

- Accepted Date: 19 Nov 2019;

- Publication Date: 26 Nov 2019.

Copyright

(C) 2019 Science Excel. This is an openaccess article distributed under the terms of the Creative Commons Attribution 4.0 International license.

\title{
A Case of medication-induced fatal fulminant liver failure in a woman with hormone-positive breast cancer
}

\author{
Nathaniel Parker ${ }^{1 *}$ and Karl Hamouche ${ }^{2}$ \\ 'Department of Internal Medicine, University of Kansas School of Medicine, Wichita, KS USA \\ 2Department of Radiology, Wesley Medical Center, Wichita, KS USA
}

\section{Case Description}

A 79-year-old female with a past medical history notable for stage IV breast cancer $(\mathrm{ER}+, \mathrm{PR}+, \mathrm{HER} 2-$ ) refractory to standard of care chemotherapy, diabetes mellitus, hyperlipidemia, and gastric esophageal reflux disease presented to the hospital with acute encephalopathy and jaundice. Recently, she had been started on palbociclib and fulvestrant. Other home medications included metformin, atorvastatin, and omeprazole. Clinical examination revealed confusion, jaundice, and generalized abdominal pain. Liver injury was evident based on lab results showing an elevated total bilirubin of 5 $\mathrm{mg} / \mathrm{dl}$, aspartate transaminase of $200 \mathrm{u} / \mathrm{l}$, alanine transaminase of $50 \mathrm{u} / \mathrm{l}$, alkaline phosphatase of $150 \mathrm{u} / \mathrm{l}$, INR of 3.8 , ammonia of $70 \mathrm{u} / \mathrm{L}$, and platelets of $35 \mathrm{x} 109 / \mathrm{L}$. Serum cirrhotic studies, acetaminophen levels, autoimmune serology and viral hepatitis workup was non-revealing. Roussel Uclaf Causality Assessment Method (RUCAM) for palbociclib was 8 (probable) [1,2]. DrugInduced Liver Injury Network (DILIN) severity for palbociclib was 5+ (fatal) [3]. Imaging studies were obtained but limited to abdominal sonography due to acute kidney injury that was noted on admission. Sonography showed a homogenous, nodular and enlarged liver surrounding extending inferiorly beyond the right kidney. Gallbladder sludge was present with borderline but nonspecific wall thickening. No was an absence of surrounding fat stranding or pericholecystic fluid. All hepatic

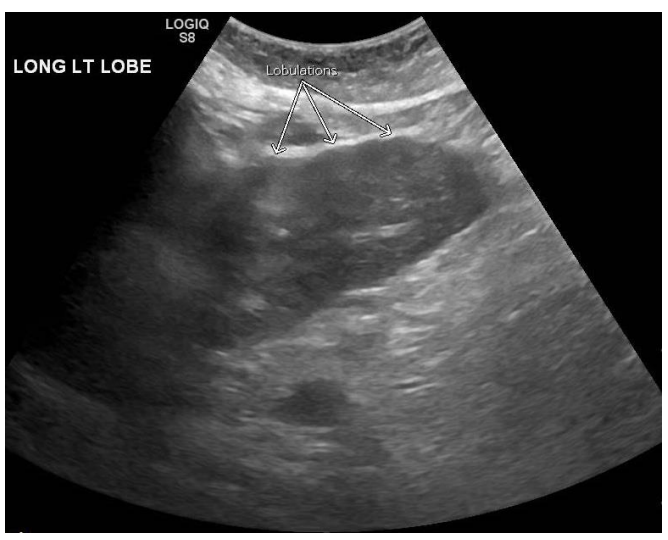

findings compared were compared to abdominal imaging studies performed two-years prior and determined to be new and significant. Despite standard of care therapies and steroids her condition deteriorated. Aspartate transaminase and total bilirubin continued to increase beyond levels greater than 10 times the upper limit of normal representing worsening liver injury. The patient subsequently received supportive treatment and was transitioned to hospice care.

Acute, or fulminant, liver failure describes the abrupt onset of hepatocellular injury and dysfunction, within 26 weeks' duration, in a patient with previously normal liver function studies. Most commonly it's associated with hepatic encephalopathy, coagulopathies, and nonspecific constitutional symptoms [4]. Importantly, liver function testing can be abnormal or unremarkable [5]. If acute liver failure is suspected a broad differential must be considered. However, medications are most commonly the culprit [5]. Although, acute liver failure is a clinical diagnosis imaging studies can be helpful to exclude other possible etiologies that may present similarly. Ultrasound remains the best initial management, despite normal imaging not being able to exclude liver injury [6]. Hepatomegaly is the most sensitive sign [7]. Commonly decreased liver echogenicity is present, which represent an early disease process. In contrast, later or more prolonged process of liver injury more commonly result in a heterogeneous echotexture [4]. A "starry sky appearance" can at

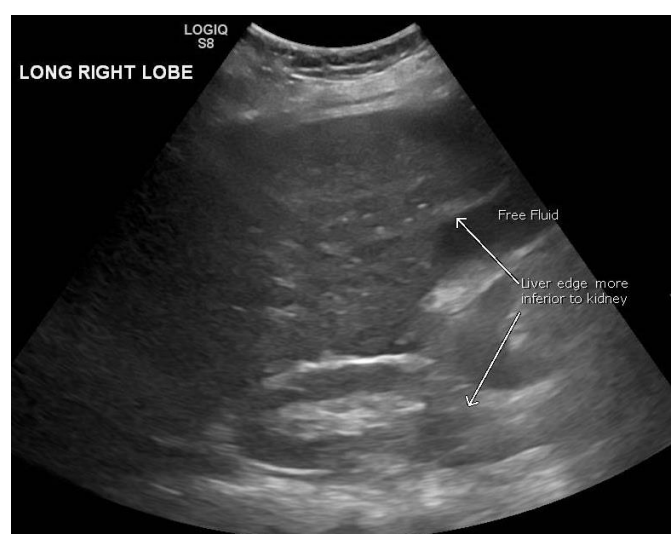

Figure 1. Ultrasound, liver. Top, homogenous liver with a grossly lobular appearance (arrows). Bottom, hepatomegaly represented by liver tip extending inferiorly beyond the level of the kidney, ascites (arrow).
Citation: Parker N, Hamouche K. A Case of medication-induced fatal fulminant liver failure in a woman with hormone-positive breast cancer. Case Rep Rev. 2019;1(1):1-2. 
times be observed due to liver parenchyma exhibiting both intermittent bright echogenic dots among a diffusely diminished parenchymal echogenicity. However, this sign has fallen out of favor due to its poor sensitivity and specificity [8]. Often contributing to equivocal workups, nonspecific hepatobiliary changes can be present or absent, such as gallbladder wall thickening and periportal edema $[9,10]$. Abdominal CT or MRI is not a first line imaging modality for acute liver failure. Typically, these more advanced imaging techniques are more clinically useful to rule out less common and more elusive etiologies, or for preoperative evaluation [5]. The patient's ultrasound findings were clinically useful in making the diagnosis and differentiating between other etiologies. Cirrhosis can present with coarse surface nodularity. Typically, a heterogeneous echotexture would be expected. Moreover, advanced cirrhosis more commonly presents as a smaller liver, not hepatomegaly [11]. For this case, the liver was enlarged and not small which can help differentiate it from cirrhosis. Acute liver failure can have features similar to cirrhosis such as nodular liver, gallbladder wall thickening and ascites. All of which were found in this patient.

\section{Disclosures}

\section{Authors' contributions}

NP and KM performed data collection and wrote manuscript. NP and KM participated in editing of the manuscript. All authors have read and approved of the article.

\section{Ethics approval and consent to participate}

The data collection on the patient had been approved by the local Ethical Committee.

\section{Consent for publication}

Written informed consent for publication of their clinical details and/or clinical images was obtained from the patient/parent/guardian/ relative of the patient.

\section{Competing interests}

The authors declare that they have no competing interests.

\section{References}

1. Danan G, Benichou C. Causality assessment of adverse reactions to drugs-I. A novel method based on the conclusions of International Consensus Meeting: Application to drug-induced liver injuries. J Clin Epidemiol 1993; 46(11):13231130.

2. Benichou C, Danan G, Flahault A. Causality assessment of adverse reactions to drugs-II. An original model for validation of drug causality assessment methods: Case reports with positive rechallenge. J Clin Epidemiol 1993; 46(11):1331-1336.

3. Hayashi PH. Drug-Induced Liver Injury Network causality assessment: Criteria and experience in the United States. Int J Mol Sci 2016; 17(2):201.

4. Ali Al-Khafaji, Su Min Cho. Acute Hepatic Failure, Fulminant Hepatic Failure, Acute Liver Failure. Cancer Therapy Advisor: Critical Care Medicine. https://www. cancertherapyadvisor.com/home/decision-support-in-medicine/critical-caremedicine/acute-hepatic-failure-fulminant-hepatic-failure-acute-liver-failure. Accessed October 12, 2019.

5. Lee WM, Stravitz RT, et al. Introduction to the revised American Association for the Study of Liver Diseases position paper on acute liver failure 2011. Hepatology. 2012;55:965-7.

6. Joshi G, Crawford KA, et al. US of Right Upper Quadrant Pain in the Emergency Department: Diagnosing beyond Gallbladder and Biliary Disease. (2018) Radiographics: a review publication of the Radiological Society of North America, Inc. 38 (3): 766-793.

7. Tchelepi H, Ralls PW, Radin R, et al. Sonography of diffuse liver disease. J Ultrasound Med. 2003;2 (9): 1023-32.

8. Heller MT, Tublin ME. The Role of Ultrasonography in the Evaluation of Diffuse Liver Disease. Radiol. Clin. North Am. 2014;52(6): 1163-1175.

9. Kurtz $A B$, Rubin CS, Cooper HS et al. Ultrasound findings in hepatitis. Radiology. 1980;136(3): 717-23.

10. Giorgio A, Amoroso P, Fico P, et al. Ultrasound evaluation of uncomplicated and complicated acute viral hepatitis. J Clin Ultrasound. 1986;14(9):675-9.

11. Romero $\mathrm{M}$, Palmer $\mathrm{SL}, \mathrm{Kahn} J \mathrm{~A}$, et al. Imaging appearance in acute liver failure: correlation with clinical and pathology findings. Dig. Dis. Sci. 2014;59(8): 1987-95. 